\title{
Desenvolvimento da Escala de Percepção de Futuro da Aposentadoria (EPFA) e Correlatos Psicossociais
}

\author{
Julia Carolina Rafalski - Universidade Federal do Espirito Santo, Vitória, Brasil \\ Alexsandro Luiz De Andrade - Universidade Federal do Espírito Santo, Vitória, Brasil
}

\begin{abstract}
Resumo
A aposentadoria é um tema em ascendência no Brasil, impulsionada por fatores demográficos, socioeconômicos e previdenciários. O processo da aposentadoria admite como primeira etapa a formação de percepções sobre a futura transição. O objetivo deste trabalho foi desenvolver e apresentar evidências de validade da Escala de Percepção de Futuro da Aposentadoria (EPFA). Foram realizados procedimentos de construção, validade de constructo e conteúdo, seguidos análise fatorial exploratória e confirmatória, além de correlações e comparações com variáveis demográficas. Participaram da pesquisa 982 trabalhadores, maioria do sexo masculino $(65,1 \%, N=640)$, com idade média de 40,1 anos ( $D P=10,5$ anos). Os resultados das aferições psicométricas confirmam a estrutura de cinco dimensões esperada, com subscalas de Percepções de Saúde, Desligamento do Trabalho, Finanças, Relacionamentos Interpessoais e Perdas na Aposentadoria. De forma geral, os resultados apontam que melhores percepções sobre o futuro na aposentadoria relacionaram-se com melhores condições contextuais de vida. São apresentados usos e limitações da medida.

Palavras-chave: aposentadoria, desenvolvimento de medida, carreira, escala psicométrica
\end{abstract}

Development of the Perceptions of Future Retirement Scale (PFRS) and Psychosocial Correlates

\begin{abstract}
Retirement is a field in ascending importance in Brazil due to demographic, socioeconomic and social security factors. The retirement process states as its first stage the perceptions about the transition. This paper aimed to develop and validate the Perceptions of Future Retirement Scale (PFRS). Development, face and construct validity assessment procedures were performed, followed by exploratory and confirmatory analysis and also comparison and correlation analysis. The participants were 982 workers, mostly male $(65.1 \%, \mathrm{~N}=640)$ with mean age of 40.1 years ( $\mathrm{SD}=10.5$ years). Results of psychometric measurements confirmed the presumed 5 factors structure, with subscales of Health Perception, Detachment from Work, Finances, Interpersonal Relationships and Losses in Retirement. In general, results show better perceptions to be related to better contextual conditions of life. Uses and limitations of PFRS are presented.

Keywords: Retirement, Scale Development, Career; Psychological Instrument
\end{abstract}

Desarrollo de la Escala de Percepción de la Futura Jubilación (EPFJ) y Conexiones Psicosociales

\begin{abstract}
Resumen
La jubilación es un tema en ascendente importancia en Brasil, impulsada por factores demográficos, socio económicos y de seguridad social. El proceso de jubilación señala como primera etapa la formación de percepciones sobre la futura transición. El objetivo de este trabajo fue desarrollar y presentar evidencias de validez de la Escala de Percepción de la Futura Jubilación (EPFJ). Se realizaron procedimientos de construcción, validez de constructo y contenido, también análisis factorial exploratorio y confirmatorio además de correlaciones y comparaciones con variables demográficas. Participaron de la investigación 982 trabajadores, la mayoría de sexo masculino $(65,1 \%, \mathrm{~N}=640)$ con edad media de 40,1 años (DP = 10,5 años). Los resultados de las mediciones psicométricas confirman la estructura de 5 dimensiones, con sub-escalas de Percepción de la Salud, Alejamiento del Trabajo, Finanzas, Relaciones Interpersonales y Pérdidas en la Jubilación. En general, los resultados señalan que una mejor percepción sobre el futuro de la jubilación se relaciona con mejores condiciones del contexto de vida. Se presentan usos y limitaciones de la medida EPFJ.
\end{abstract}

Palabras-clave: Jubilación; Desarrollo de la Medida; Carrera; Escala Psicométrica

\section{Introdução}

A transição para a aposentadoria é um assunto de ascendente interesse para pesquisadores e trabalhadores, impulsionado principalmente por fatores demográficos de crescimento da expectativa de vida, fatores socioeconômicos e previdenciários (Szinovacz, 2003). Uma visão tradicional da aposentadoria sugere que essa fase começa com o fim do trabalho remunerado e termina com a morte do indivíduo (Beehr \& Bowling, 2012), estendendo-se por um período de duração variável tendo em vista a idade de cessação das atividades profissionais e a longevidade esperada em cada país (Shultz \& Wang, 2011).

Segundo as concepções de Adams e Rau (2011) e Wang (2007), pode-se compreender diferentes estágios da transição trabalho-aposentadoria, abrangendo questões antecedentes e consequentes do "se aposentar". 
A formação de percepções sobre o futuro na aposentadoria é o primeiro de cinco passos que representam o processo de transição entre "ser trabalhador" e "ser aposentado", com etapas seguintes de "decisão", "planejamento", "ajustamento" e "satisfação" (Adams\& Rau, 2011; Wang, 2007).

Um entendimento holístico e compreensivo do processo de transição considera relevantes as dimensões que circundam o indivíduo, tais como a saúde, o trabalho, os relacionamentos, os papéis sociais e as finanças (Wang \& Shultz, 2010). Assim, o indivíduo que se preocupa com as diferentes esferas da vida consegue se inserir de forma mais completa no momento de transição e na experiência de ser aposentado, tendo maior qualidade nas cinco etapas do processo (Lee \& Law, 2004).

No Brasil, pesquisadores têm concentrado seus esforços na compreensão das representações e opiniões sobre a aposentadoria em estudos de maioria qualitativa (p. ex., Bressan, Mafra, França, Melo, \& Loreto, 2013; Kegler \& Macedo, 2015). Estudos sobre planejamento para a aposentadoria com abordagem quantitativa ( $p$. ex., Leandro-França, Murta, \& Villa, 2014) ainda são minoria nas publicações em periódicos científicos em relação às demais publicações na área e estudos quantitativos que abordem a primeira fase do processo de transição não foram encontrados, ainda que a temática das percepções não seja recente no estudo da Psicologia.

\section{Percepção de Futuro e as Dimensões da Aposentadoria}

Perceber é um ato natural e cotidiano, que ocorre a todo momento, suscitado pelo ambiente, pelas pessoas e pelos objetos que circundam o ser humano. De acordo com Fiske e Taylor (2013), as percepções são adaptativas e sofrem influência do contexto de vida do indivíduo e das informações que este recebe. Fazem parte da construção da cognição social, que se refere aos processos por meio dos quais as pessoas compreendem e explicam acontecimentos a si mesmas e ao demais com quem interagem (Trócolli, 2011).

Atitudes e percepções se estendem em uma linha temporal. Perceber tanto precede quanto sucede as atitudes (Fiske \& Taylor, 2013). É necessário obter informações sobre as condições em que essa ocorrência se situa, proceder um julgamento sobre as informações e então se expressar, baseando-se no contexto e em sua opinião (Macrae \& Bodenhausen, 2000). A perspectiva de tempo, na maioria das vezes, atua como um processo inconsciente e utiliza as experiências pessoais e sociais do indivíduo para fornecer significado e ordem a eventos passados e futuros (Zimbardo \& Boyd, 1999).
No contexto da aposentadoria, atitudes e percepções influenciam sequentes comportamentos de planejamento (Noone, Stephens, \& Alpass, 2009). Percepções sobre a aposentadoria demonstraram ter um impacto significativo na preparação financeira, de estilo de vida e psicossocial (Noone, Stephens, \& Alpass, 2010).

A partir da revisão bibliográfica em diferentes periódicos com publicações sobre o tema aposentadoria, é possível constatar o agrupamento de temáticas que dizem respeito ao assunto. São elas: 1) saúde, 2) finanças, 3) trabalho, 4) relacionamentos e 5) uso do tempo. Essas dimensões, aliadas às variáveis demográficas de contexto (p. ex., idade, escolaridade, renda, entre outras), auxiliam a tornar a compreensão da aposentadoria mais complexa e abrangente.

Segundo Zanelli (2012), a percepção de se aposentar envolve percepções positivas do bem-estar físico, emocional e comportamental, além de componentes do trabalho e dos relacionamentos. Indivíduos com a maioria de sua rede formada pelo círculo profissional tendem a perceber a aposentadoria como mais solitária e isoladora (França, Menezes, \& Siqueira, 2012; Petkoska \& Earl, 2009), podendo impactar em sua percepção de si mesmo (Hershey \& Henkens, 2014). Fatores econômicos, como a pensão e os planos de economia para a aposentadoria influenciam a decisão e a preparação para essa fase da vida: quanto mais estável a situação financeira menor é a idade esperada para se aposentar e menor é a expectativa de se realizar atividades de cunho profissional após a aposentadoria (Hira, Rock, \& Loibl, 2009; Pires et al., 2013).

A saúde psicossocial também tem um impacto considerável na aposentadoria. Segundo Leung e Earl (2012), a saúde é um dos recursos mais necessários para promover ajustamento na aposentadoria, bem como a autonomia e o bem-estar (Leandro-França et al., 2014). Segundo Juvenal, Viana, Vasconcelos e Santos (2015), as percepções podem também se mostrar contraditórias, na medida em que os trabalhadores contrapõem o ponto positivo do descanso e da recompensa com o ponto negativo da incerteza e das perdas sócias. No geral, Maggiori, Nihil, Froidevaux e Rossier (2013) observaram que, conforme os trabalhadores se aproximam da aposentadoria, suas percepções sobre o evento se tornam mais positivas, porém menos concretas.

Gutierrez e Hershey (2014), em estudo com 90 adultos de meia idade $(M=49,09$ anos, $D P=7,91$ anos) e 1560 estudantes de graduação $(M=19,49$ anos, $D P=2,08$ anos), buscaram identificar a possibilidade 
de diferenças entre grupos de idade nas expectativas de satisfação na aposentadoria futura e os fatores psicológicos preditores dessas percepções de futuro quanto à satisfação com a vida. Para tanto, utilizaram a Escala de Satisfação com a Vida na Aposentadoria (Satisfaction With Life in Retirement Scale - SWLRS).

Quando comparando as idades, o grupo de estudantes demonstrou percepções de aposentadoria futura significativamente mais positivas que o grupo de adultos de meia idade. O modelo de path analysis elaborado pelos autores foi efetivo na identificação de fatores psicológicos responsáveis pelas percepções dos indivíduos sobre a aposentadoria: influência dos pais no comportamento de economia, perspectiva de tempo futuro, conhecimento em finanças e tolerância ao risco financeiro - todos os possíveis preditores utilizados na pesquisa. Para os autores, a diferença de média para os adultos de meia idade pode significar que eles entendem a aposentadoria com maior olhar para as nuances, considerando que a satisfação com a aposentadoria no futuro também dependerá da habilidade de garantir a saúde, manter vínculos sociais fortes e o vigor físico e intelectual.

A partir das considerações anteriores, a pouca presença de estudo sobre o fenômeno da aposentadoria no Brasil (Zanelli, 2012) e principalmente a carência de instrumentos psicométricos para avaliação desses aspectos no contexto brasileiro, este artigo tem como objetivo apresentar as etapas de construção e verificação de propriedades psicométricas de uma escala desenvolvida para avaliar percepções futuras de aposentadoria entre trabalhadores brasileiros. Em seguida, procura-se compreender as relações entre as percepções de trabalhadores sobre seu futuro na aposentadoria e outros constructos psicossociais (centralidade do trabalho, motivos para aposentar e saúde).

\section{Delineamento Metodológico}

\section{Procedimentos para o Desenvolvimento da Escala}

A realização da pesquisa foi avaliada e aprovada pelo Comitê de Ética da Universidade Federal do Espírito Santo sob o número 473.624. A construção dos itens da EPFA tomou como base estrutura compreensiva do planejamento da aposentadoria (Wang, 2007; Wang \& Shultz, 2010) para abordar percepções equivalentes às dimensões já citadas. Foram elaborados 10 itens para cada uma das dimensões a seguir: a) desligamento do trabalho; b) relacionamentos interpessoais, c) segurança financeira; d) uso do tempo e; e) saúde e bem-estar, a qual contou com 11 itens durante a primeira etapa de desenvolvimento, resultando um total de 51 itens preliminares.

Os 51 itens preliminares foram avaliados quanto a critérios de pertinência ao fator de criação e semântica por cinco doutores em Psicologia com experiência em construção de instrumentos e/ou conhecimento na área de aposentadoria. Tomadas as primeiras considerações por parte dos avaliadores especialistas, seis trabalhadores com idades acima de 35 anos e atuantes em empresas públicas e privadas foram convidados a responderem à escala.

\section{Análise de Conteúdo de Juízes}

Os itens confeccionados foram organizados de forma aleatória na versão preliminar da escala. Utilizou-se o critério de $80 \%$ de concordância entre os juízes para a manutenção de itens (Hulley et al., 2003). As subscalas Segurança Financeira e Relacionamentos Interpessoais mantiveram seus 10 itens iniciais. A subscala Saúde e Bem-Estar passou a contar com nove itens, ao invés de 11 itens iniciais, tendo sido um excluído devido a discordância entre juízes e o outro movido para a escala de uso do tempo. A escala desligamento do trabalho passou a contar com sete itens, tendo sido três excluídos pela validade de face devido a dubiedade em relação às dimensões. A subscala uso do tempo obteve três itens excluídos e um item adicionado por transferência da subscala de saúde e bem-estar, contando ao final com oito itens. Após a análise, a versão inicial da Escala de Percepção Futura da Aposentadoria contou com 44 itens alocados nas cinco dimensões previamente agrupadas.

\section{Análise Semântica}

A versão de 44 itens foi aplicada individualmente em seis participantes utilizando um questionário que também continha questões demográficas e outros instrumentos. Os participantes foram convidados a opinar sobre a redação e a compreensibilidade dos itens e da instrução. Não houve relato de dificuldades quanto aos itens ou à instrução e o questionário se manteve inalterado.

\section{Levantamento dos Indicadores Empiricos de Validade e Precisão}

\section{Participantes}

Nessa etapa, a amostra geral $(N=982)$ foi randomicamente divida em dois grupos (G1 = amostra fatorial e G2 = amostra confirmatória) contendo 491 
participantes cada. Foram atendidos critérios de equivalência segundo as variáveis de idade (G1, $M=40,3$; $D P=10,5 ; \mathrm{G} 2, M=39,6 ; D P=10,5)$, sexo (G1 e $\mathrm{G} 2$, $65,3 \%$ homens e $34,7 \%$ mulheres) e tipo de vínculo empregatício (G1, público $=69,9 \%$; privado $=30,1 \%$; $\mathrm{G} 2$, público $=71,6 \%$; privado $=28,4 \%$ a fim de manter a equiparação entre as amostras.

\section{Procedimentos de Coleta e Análise de Dados}

A coleta de dados foi realizada após a obtenção de autorização por parte de empresas públicas e privadas de um estado na região Sudeste, que foram convidadas a participar do projeto. Os representantes de Recursos Humanos de 25 organizações de pequeno, médio e grande porte foram contatados para a apresentação do projeto de pesquisa e solicitação de parceira e somente oito organizações retornaram contato com interesse. Os trabalhadores foram convidados a responder o questionário voluntariamente, em versão impressa ou em um sítio de internet, de acordo com a preferência da empresa - os questionários impressos foram entregues a todos os funcionários com idades acima de 40 anos em envelopes selados para serem devolvidos ao $\mathrm{RH}$ depois de respondidos, sem identificação; para os questionários online, a pesquisa foi encaminhada via e-mail para todos os funcionários das empresas pelo $\mathrm{RH}$. Todos receberam Termos de Consentimento Livre e Esclarecido em duas vias na versão impressa ou online, além de informações gerais sobre os objetivos da pesquisa e sobre a liberdade para desistirem a qualquer momento sem prejuízos. Os questionários que apresentavam menos de $10 \%$ de dados omissos foram considerados para análise.

Os dados foram analisados com o auxílio do pacote estatístico SPSS e AMOS. Para a verificação da estrutura dimensional, recorreu-se a análise dos componentes principais $(P C A)$ e, para levantamento dos indicadores de validade de constructo, realizou-se a fatorial exploratória $(P A F)$. Utilizou-se o teste alfa de Cronbach para avaliação da precisão das subscalas resultantes. Para a análise confirmatória, foram utilizados os seguintes indicadores: Qui-quadrado ponderado, obtido pela razão do Qui-quadrado por graus de liberdade $\left(\chi^{2 / g}\right)$, menor que 3; o goodness of fit index (GFI) e o comparative fit index (CFI), valores próximos a 1; e o root mean square error of approximation (RMSEA), valor inferior a 0,08 (Byrne, 2010).

\section{Indicadores de Fatorabilidade e Validade}

A análise dos componentes principais demonstrou boa fatorabilidade da matriz de dados. O KMO obteve valor favorável de 0,88 , e o teste de esfericidade de Bartlett foi significativo $\left[\chi^{2}(946)=8893,0, p=\right.$ $0,000]$. Para decisão do número de fatores, optou-se pelo método de análise do scree plot. Os dados sugeriram a estrutura de cinco dimensões, conforme se previu nas etapas anteriores.

Por meio do método dos componentes principais, extraiu-se a solução de cinco fatores, a qual explicou $46,2 \%$ de variância dos dados. Utilizou-se a rotação oblíqua, que assume relação entre fatores (Dancey \& Reidy, 2006), proporcionando bom ajuste a matriz de dados. $\mathrm{Na}$ solução final da medida, optou-se por manter apenas os cinco itens de maior carga em cada subscala e conservar a equivalência numérica entre as dimensões. A escala em sua versão final com 25 itens, cargas fatoriais e índices de consistência interna é apresentada na Tabela 1.

A solução de cinco fatores encontrada corrobora o número esperado no momento de construção da escala e as dimensões são equivalentes nos constructos Saúde, Desligamento do Trabalho, Relacionamentos Interpessoais e Finanças, respectivamente os fatores I, II, III e V. Contudo, a dimensão Uso do Tempo não manteve a estrutura planejada. Itens inicialmente designados para esse fator se misturaram a itens de contexto negativo presentes na dimensão Relacionamentos Interpessoais, gerando a subscala denominada Perdas da Aposentadoria, fator IV.

O fator I, denominado Percepções de Saúde, abarca percepções acerca do bem-estar ligado à saúde na aposentadoria, realização de atividades para se manter saudável e às dificuldades relacionadas à falta de saúde, com dois itens (números 8 e 41) inversos. Maiores escores indicam percepções mais positivas com relação à saúde na aposentadoria. A subscala possui alfa de 0,85 .

O fator II foi denominado Desligamento do Trabalho. Essa dimensão caracteriza opiniões relativas à saída do trabalho e a percepção de facilidade ou dificuldade de deixar de exercer atividades profissionais. Escores mais elevados indicam percepção mais positiva da saída do mercado e do ingresso na aposentadoria. Possui alfa de 0,79 e um item inverso (número 14).

O fator III foi denominado Relacionamentos Interpessoais e inclui aspectos de convivência com familiares e colegas de trabalho, habilidade de fazer e manter amigos, e a qualidade geral dos relacionamentos. Maiores escores indicam a percepção de que os relacionamentos na aposentadoria são positivos e podem continuar sem interferência da saída do trabalho. $\mathrm{O}$ alfa da subscala é 0,70 . 
Tabela 1

Estrutura Fatorial da Escala de Percepção Futura da Aposentadoria (EPFA)

\begin{tabular}{|c|c|c|c|c|c|}
\hline Itens & I & II & III & IV & h2 \\
\hline $\begin{array}{l}\text { Terei dificuldades para viver minha aposentadoria pela falta de } \\
\text { saúde. }\end{array}$ & 0,75 & & & & 0,59 \\
\hline $\begin{array}{l}\text { Penso que terei saúde o suficiente para ser ativo na minha } \\
\text { aposentadoria. }\end{array}$ & $-0,73$ & & & & 0,65 \\
\hline Penso que envelhecerei com boa saúde. & $-0,72$ & & & & 0,69 \\
\hline Acredito que terei boa saúde na aposentadoria. & $-0,72$ & & & & 0,62 \\
\hline Dificilmente terei uma aposentadoria saudável. & 0,61 & & & & 0,49 \\
\hline $\begin{array}{l}\text { Poder parar de trabalhar é o que mais me alegra quando penso } \\
\text { em me aposentar. }\end{array}$ & & $-0,77$ & & & 0,59 \\
\hline $\begin{array}{l}\text { Para mim, parar de trabalhar é mais um ganho que uma perda na } \\
\text { aposentadoria. }\end{array}$ & & $-0,77$ & & & 0,59 \\
\hline Penso que sair do trabalho é o maior ganho da aposentadoria. & & $-0,63$ & & & 0,41 \\
\hline Acredito que terei facilidade para me desligar do trabalho. & & $-0,62$ & & & 0,52 \\
\hline Mesmo estando cansado, ficarei triste por parar de trabalhar. & & 0,58 & & & 0,49 \\
\hline $\begin{array}{l}\text { Acredito que terei bons relacionamentos interpessoais ao me } \\
\text { aposentar. }\end{array}$ & & & 0,62 & & 0,49 \\
\hline $\begin{array}{l}\text { Mesmo não convivendo mais diariamente no trabalho, acredito } \\
\text { que manterei os amigos que fiz no ambiente profissional ao } \\
\text { aposentar. }\end{array}$ & & & 0,58 & & 0,36 \\
\hline $\begin{array}{l}\text { Acredito que conseguirei fazer amigos quando me aposentar } \\
\text { com a mesma facilidade que tenho hoje. }\end{array}$ & & & 0,56 & & 0,43 \\
\hline Espero manter meus amigos quando me aposentar. & & & 0,53 & & 0,30 \\
\hline $\begin{array}{l}\text { Quando me aposentar, acredito que poderei conviver e me } \\
\text { aproximar mais dos meus familiares. }\end{array}$ & & & 0,51 & & 0,31 \\
\hline Penso que me sentirei muito sozinho durante a aposentadoria. & & & & 0,59 & 0,53 \\
\hline Será difícil lidar com a falta de amigos na aposentadoria. & & & & 0,51 & 0,39 \\
\hline $\begin{array}{l}\text { Prevejo que perderei muitos de meus amigos quando me } \\
\text { aposentar. }\end{array}$ & & & & 0,51 & 0,34 \\
\hline $\begin{array}{l}\text { Será difícil administrar todo o tempo livre que terei quando me } \\
\text { aposentar. }\end{array}$ & & & & 0,47 & 0,3 \\
\hline $\begin{array}{l}\text { Dificilmente terei atividades que preencham meu tempo livre } \\
\text { quando me aposentar. }\end{array}$ & & & & 0,45 & 0,33 \\
\hline
\end{tabular}

Nota. h2 = Comunalidades. Principal Axis Fatorial, método direct oblimin. I: Percepções de Saúde; II: Desligamento do Trabalho; III: Relacionamentos Interpessoais; IV: Perdas da Aposentadoria. 
Tabela 1

Estrutura Fatorial da Escala de Percepscão Futura da Aposentadoria (EPFA) (Continuação)

\begin{tabular}{|c|c|c|c|c|c|c|}
\hline Itens & I & II & III & IV & $\mathrm{V}$ & h2 \\
\hline $\begin{array}{l}\text { Penso que terei uma vida confortável, levando em consideração a } \\
\text { pensão que receberei. }\end{array}$ & & & & & $-0,68$ & 0,57 \\
\hline $\begin{array}{l}\text { Provavelmente terei que buscar novos meios de obter renda para } \\
\text { me manter estável financeiramente na aposentadoria. }\end{array}$ & & & & & 0,68 & 0,53 \\
\hline $\begin{array}{l}\text { Mesmo com uma pensão menor que meu salário atual, penso que } \\
\text { terei estabilidade financeira na aposentadoria. }\end{array}$ & & & & & $-0,67$ & 0,47 \\
\hline $\begin{array}{l}\text { Ainda que a pensão seja pequena, creio que terei uma } \\
\text { aposentadoria financeiramente agradável. }\end{array}$ & & & & & $-0,66$ & 0,59 \\
\hline Acredito que será difícil viver com uma pensão. & & & & & 0,62 & 0,46 \\
\hline Alfa de Cronbach & 0,85 & 0,79 & 0,70 & 0,70 & 0,81 & \\
\hline
\end{tabular}

Nota. h2 = Comunalidades. Principal Axis Fatorial, método direct oblimin. I: Percepções de Saúde; II: Desligamento do Trabalho; III: Relacionamentos Interpessoais; IV: Perdas da Aposentadoria. V: Percepções Financeiras

O fator IV, denominado de Perdas da Aposentadoria, expõe percepções de dificuldade na vivência da aposentadoria, tanto em relação ao mau aproveitamento do tempo disponível quanto a sentimentos de solidão e abandono. Pessoas com escores mais elevados mostram perceber mais perdas na aposentadoria, considerando-a mais triste e desamparada. Possui alfa de 0,70 .

$\mathrm{O}$ quinto e último fator recebeu o nome de Percepções Financeiras. Essa dimensão explora a estabilidade financeira, aumento de gastos e preocupações relativas à obtenção e complementação de renda na aposentadoria. Maiores escores indicam participantes que percebem maior sucesso e estabilidade financeira. O alfa da subscala é de 0,81 e os itens 10 e 38 são invertidos.

Com a segunda parte da amostra (G2), partiu-se para o teste do modelo fatorial resultante da escala de percepção futura de aposentadoria. A análise fatorial confirmatória da escala foi conduzida com o conjunto de 25 itens dispostos num modelo único de cinco fatores intercorrelacionados. Os indicadores de ajuste do modelo não foram plenamente satisfatório $\left[\chi^{2}=\right.$ $1072,876, g l=265(p<0.0001), \chi^{2} / g l=4,049$, RMR $=0,108$, GFI $=0,837$, AGFI $=0,801$, CFI $=0,829$, RMSEA $(90 \% C I)=0,79(0,74-0,84]$.

A partir desses resultados, realizaram-se alterações segundo os índices de modificação para melhora nos indicadores. Observaram-se valores elevados de resíduos padronizados entre os pares de parâmetros de erros de alguns itens. Foram adotadas modificações estabelecendo uma covariância entre erros dos itens. O novo modelo foi testado e apresentou indicadores de ajustes mais próximos do aceitável: $[\chi 2=945,872, g l=$ $261(p<0,001), \chi^{2} / g l=3,62, \mathrm{RMR}=0,10$; GFI $=0,86$, AGFI $=0,83$, CFI $=0,86$, RMSEA $(90 \% C I)=0,073$ $(0,068-0,078)]$.

Rede Nomológicado Construto Percepşão de Futuro da Aposentadoria

\section{Participantes}

Os participantes dessa etapa compreendem a totalidade dos presentes na etapa de levantamento de indicadores de validade e precisão, sendo a amostra de 982 trabalhadores. A média de idade da amostra foi de 40,1 anos ( $D P=10,5$ anos), com maioria dos respondentes do sexo masculino $(65,1 \%, N=640)$ e com vínculo empregatício em empresas públicas $(68,7 \%$, $N=694)$. A maioria dos participantes reside em um estado da região Sudeste do Brasil (91,7\%), possui pelo menos Ensino Superior completo $(74,8 \%)$ e é casada $(68,9 \%)$. A média do tempo de experiência profissional da amostra é de 18,26 anos ( $D P=10,8$ anos).

\section{Instrumentos}

Foi estruturado um questionário tipo survey contendo as seguintes medidas: a) Escala de Percepção de Futuro da Aposentadoria (EPFA), avaliada em 
sua versão de 25 itens. Os alfas de Cronbach de cada uma das dimensões estão disponíveis no Estudo 1; b) Escala adaptada e reduzida de Saúde SF-36 - SF-36 Health Status Questionnaire (Ware \& Sherbourne, 1992). Foram utilizadas as escalas de Saúde Geral (cinco itens, $\alpha=0,74$ ) e a escala de Saúde para o Trabalho (quatro itens, $\alpha=0,72$ ) (Rafalski \& De Andrade, 2014); c) Escala de Motivos para se Aposentar - Reasons for Retirement Questionnaire (Robinson, Demetre, \& Corney, 2010). Adaptada para o português, dividida em duas dimensões: Controle Pessoal (seis itens, $a=0,71$ ) e Circunstâncias Externas (três itens, $a=0,70$ ) (Rafalski \& De Andrade, 2014); d) Escala de Centralidade do Trabalho - Work Centrality Scale (Paullay, Alliger, \& Stone-Romero, 1994). Adaptada para o português (Rafalski \& De Andrade, 2014), possui 12 itens ( $a=$ $0,83)$ e é unifatorial. A escala demonstrou bons índices de validade e confiabilidade; e) questionário sociodemográfico. Continha questões para identificação da amostra, como idade, sexo, tipo de empresa em que atua, renda esperada para a aposentadoria e idade em que acredita que se aposentará.

\section{Procedimentos de Coleta e Análise de Dados}

Os procedimentos para coleta de informações foram os mesmos do Estudo 1. Quanto à análise de dados, realizou análises de correlação com as variáveis coletadas na amostra do estudo e análises de comparação de médias entre grupos (acreditam que aposentarão com mais de 60 anos x com menos de 59 anos, e empresas públicas $\mathrm{x}$ empresas privadas).

\section{Indicadores de Validade Convergente e Correlações}

Analisaram-se as correlações entre os constructos da EPFA e os dados demográficos de idade e sexo e os constructos de Saúde, de Motivos para se Aposentar e Centralidade do Trabalho. Os resultados dessas análises podem ser observados na Tabela 2. Para exposição dos dados, optou-se por apresentar apenas com correlações acima de 0,3 , que apresentam maior força e impacto nos resultados (Dancey \& Reidy, 2006).

As cinco dimensões foram significativamente correlacionadas entre si, com exceção da relação entre Percepção de Saúde e Desligamento do Trabalho. As correlações entre Perdas da Aposentadoria e as quatro demais subscalas foram negativas, indicando que maior escore nas demais subscalas retratam a percepção de menos perdas associadas com a entrada na aposentadoria. Foram verificadas correlações positivas e significativas entre o constructo Percepção de Saúde e Saúde Geral da SF-36 ( $r=0,57)$, indicando que percepções futuras mais positivas se ligam à compreensão de maior qualidade na vida presente. O constructo Desligamento do Trabalho se correlacionou negativamente

Tabela 2

Correlaçoes entre Percepcỗes Futuras sobre Aposentadoria e Dados Demográficos

\begin{tabular}{|c|c|c|c|c|c|c|c|c|c|c|c|}
\hline Variáveis & 1 & 2 & 3 & 4 & 5 & 6 & 7 & 8 & 9 & 10 & 11 \\
\hline 1. PS & - & & & & & & & & & & \\
\hline 2. DT & 0,00 & - & & & & & & & & & \\
\hline 3. RI & $0,40 * *$ & $0,09 * *$ & - & & & & & & & & \\
\hline 4. PA & $-0,38^{* *}$ & $-0,21 * *$ & $-0,38^{* *}$ & - & & & & & & & \\
\hline 5. PF & $0,50^{* *}$ & 0,02 & $0,21 * *$ & $-0,22^{* *}$ & - & & & & & & \\
\hline 6. Idade & 0,03 & $-0,10 * *$ & 0,02 & $-0,05$ & $0,07 *$ & - & & & & & \\
\hline 7. Sexo & $0,14 * *$ & $-0,05$ & 0,00 & $-0,08^{*}$ & $0,06^{*}$ & $-0,03$ & - & & & & \\
\hline 8. $\mathrm{CiE}$ & $-0,19 * *$ & 0,09 & $-0,04$ & $0,14^{* *}$ & $-0,18^{* *}$ & $-0,17 * *$ & 0,01 & - & & & \\
\hline 9. $\mathrm{CoP}$ & $0,12^{* *}$ & $-0,30 * *$ & $0,06^{*}$ & 0,00 & $0,11 * *$ & $0,15^{* *}$ & $-0,00$ & $-0,41 * *$ & - & & \\
\hline 10. SG & $0,57 * *$ & $-0,04$ & $0,26 * *$ & $-0,23^{* *}$ & $0,27 * *$ & $-0,07^{*}$ & 0,06 & $-0,19 * *$ & $-0,17^{* *}$ & - & \\
\hline 11. ST & $0,23 * *$ & $-0,09 * *$ & $0,17 * *$ & $-0,08^{*}$ & $0,13 * *$ & $0,07 *$ & $-0,00$ & $0,08^{*}$ & $-0,12^{*}$ & $0,33 * *$ & - \\
\hline 12. Cent & 0,01 & $-0,51 * *$ & 0,02 & $0,21 * *$ & $0,11 * *$ & $0,25^{* *}$ & $-0,03$ & $-0,07 *$ & $0,23^{* *}$ & 0,04 & 0,05 \\
\hline
\end{tabular}

Nota. ${ }^{*} p<0,005,{ }^{* *} p<0,001$. PS $=$ percepção de saúde; DT $=$ desligamento do trabalho; RI = relacionamentos interpessoais; PA $=$ perdas da aposentadoria; $\mathrm{PF}=$ percepções financeiras; $\mathrm{CiE}=$ circunstâncias externas; $\mathrm{CoP}=$ controle pessoal; $\mathrm{SG}=$ saúde geral; $\mathrm{ST}=$ saúde do trabalho; Cent $=$ centralidade no trabalho. 
com Controle Pessoal $(r=-0,30)$ e Centralidade do Trabalho $(r=-0,51)$. Assim, trabalhadores que antecipam maiores dificuldade para se desligar de seu emprego são os que consideram o trabalho mais importante dentre as demais dimensões de vida e tendem a não considerar seus interesses externos ao trabalho no momento de aposentar.

Para compreender as diferenças entre percepção futura de aposentadoria e idade planejada para aposentar, corroborando também para indicadores sobre a validade discriminante da medida, realizou-se uma ANOVA com as dimensões da EPFA. A idade em que os participantes acreditam que se aposentarão foi recodificada para "acima de 60 anos $=1$ " e "abaixo de 59 anos $=2$ ". Utilizou-se a idade de 60 anos como corte por esta ser a idade em que os indivíduos são considerados idosos pelo Estatuto do Idoso (Brasil, 2009). Foram encontradas diferenças estatisticamente significativas entre os grupos para as dimensões de Desligamento do Trabalho $[F(1,957)=26,092, p<0,001]$ e Relacionamentos Interpessoais, $[F(1,957)=12,410, p<0,001]$.

Em ambas as dimensões, o grupo de participantes que acreditam que se aposentarão com menos de 59 anos $(M=3,29, D P=0,90$ e $M=3,95, D P=0,67$, respectivamente Desligamento e Relacionamentos) possuíram percepções mais positivas quanto a facilidade de deixar o trabalho e de manter seus relacionamentos interpessoais que os participantes que acreditam que se aposentarão com mais de 60 anos $(M=2,99, D P$ $=0,95$ e $M=3,79, D P=0,68$, respectivamente $\mathrm{DT}$ e RI). É possível supor que por esperarem se aposentar mais cedo, os indivíduos terão mais disposição e maior interesse em buscar novas atividades, fontes de prazer e relacionamentos; em contrapartida, os que esperam se aposentar mais tarde podem já por esse motivo evidenciar dificuldades em se desligar do trabalho, podendo ter um círculo menor de convivência e, por isso, percepção futura de menos relacionamentos.

Os resultados para as dimensões Saúde $[F(1,957)$ $=0,328, p=0,567]$, Perdas na Aposentadoria $[F(1,957)$ $=1,137, p=0,287]$ e Finanças $[F(1,957)=1,214, p$ $=0,271]$ não foram estatisticamente significativos. Percebe-se que independentemente da idade em que acreditam que se aposentarão, os participantes mantem suas percepções de saúde, finanças e perdas futuras, não sendo um fator de interferência para apontar que vislumbram diferenças em sua saúde, riqueza material ou aproveitamento do tempo e dos amigos.

Diante da expectativa que o tipo de vínculo que os participantes possuem seja um fator de interferência no modo como vislumbram a aposentadoria, foi realizada uma ANOVA para avaliar diferenças entre trabalhadores de empresas públicas (Grupo 1) e privadas (Grupo 2) quanto às dimensões de percepção futura de aposentadoria. Para conservar a homogeneidade, optou-se por igualar as amostras, procedendo uma exclusão aleatória de participantes do Grupo $1(N$ inicial $=694)$ até que ambos os grupos contassem com o mesmo número de participantes $(N=256)$.

Os resultados indicaram diferenças estatisticamente significativas entre as amostras apenas para as dimensões Desligamento do Trabalho $[F(1,510)=$ 15.714; $p<0,001]$ e Perdas na Aposentadoria $[F(1,510)$ $=7,443 ; p=0,007]$. Participantes de empresas públicas $(M=3,22 ; D P=0,99)$ possuem melhores percepções que os de empresas privadas $(M=2,91 ; D P=0,78)$ quanto ao desligamento do trabalho e menores percepções de perdas na aposentadoria $(M=2,07 ; D P=0,78)$ que os de empresas privadas $(M=2,24 ; D P=0,62)$, se mostrando menos suscetíveis a sentimentos de tristeza ao se desligarem, percebendo menos dificuldades para deixar de exercer atividades profissionais e menos perdas advindas do momento da aposentadoria.

Os resultados das dimensões de Saúde $[F(1,510)=$ $0,049 ; p=0,824]$, Relacionamentos $[F(1,510)=0,075$; $p=0,785]$ e Finanças $[F(1,510)=0,757 ; p=490]$ não foram estatisticamente significativos. Isso aponta que a questão burocrática que liga os indivíduos ao trabalho (desligamento e perdas advindas da saída) possui maior relevância que variáveis estritamente pessoais quanto à percepção de futuro na aposentadoria para trabalhadores de empresas públicas.

\section{Discussão}

Foi apresentado acima o processo que culminou no levantamento dos indicadores de validadeda Escala de Percepção de Futuro da Aposentadoria. Os resultados das aferições analíticas reforçam a estrutura fatorial de cinco dimensões sugerida pela abordagem compreensiva do fenômeno (Wang \& Shultz, 2010), ainda que com modificações. A junção de itens das escalas iniciais de uso do tempo e relacionamentos e os itens com conotação negativa sobre aposentadoria formaram a subscala de Perdas da Aposentadoria, que dá ênfase às dificuldades percebidas para o futuro. O surgimento dessa subscala, que não era esperada, mostra a relevância das percepções negativas e do receio em imaginar sua futura aposentadoria por parte da amostra (Zanelli, 2012). 
Não se deve, contudo, desconsiderar a importância do desenvolvimento de novas atividades devido à exclusão parcial da dimensão de uso do tempo. A dificuldade de preencher o tempo e de encontrar atividades satisfatórias atua como um reforçador da necessidade de apresentar alternativas de uso do tempo para esse momento da aposentadoria (Shultz, Morton, \& Weckerle, 1998). Participantes com escores altos na dimensão de Perdas da Aposentadoria aparentam terem melhores estratégias para traçar alternativas de atividades e novos papéis sociais no momento que se aposentarem, incluindo atividades ligadas ao bem-estar, que gerem renda ou que sejam voluntárias (França \& Vaughan, 2008).

Utilizando um conjunto semelhante de dimensões como base para um trabalho de intervenção no planejamento para aposentadoria, Leandro-França, Murta e Villa (2014) conseguiram verificar mudanças no comportamento de planejamento em servidores públicos no que diz respeito ao investimento ocupacional-social e ao investimento em autonomia e bem-estar com a Escala de Mudança em Comportamento de Planejamento da Aposentadoria (EMCPA). A escala Fatores-Chave para o Planejamento da Aposentadoria (KFRP - Key Factors on Retirement Planning) de França e Carneiro (2009) também adota dimensões semelhantes: bem-estar pessoal e social, fatores de risco ou de sobrevivência, início de uma nova carreira e relacionamentos familiares, mostrando a importância dos fatores na compreensão da aposentadoria.

Como uma medida que representa o primeiro estágio do processo da aposentadoria, a EPFA contribui para o conhecimento das percepções sobre a transição, explorando-as de forma compreensiva. A tomada de consciência sobre as percepções indica a fase de contemplação que, segundo Leandro-França, Murta e Villa (2014), pode durar um longo período.

Os resultados deste trabalho podem ser úteis para estabelecer linhas de base e parâmetros comparativos em intervenções de grupo para preparação para a aposentadoria. Ampliar positivamente as percepções da aposentadoria pode incitar trabalhadores a escolherem se aposentar por motivos mais ligados ao descanso e ao interesse em sair do trabalho. Dado que as percepções podem mudar e se atualizar com base na obtenção de informações, a EPFA pode atuar como meio de medir o desenvolvimento das concepções dos trabalhadores sobre as esferas da aposentadoria, sendo um meio confiável para acessar tais indicadores em pesquisas/ diagnósticos organizacionais.
Entre as limitações da primeira etapa de desenvolvimento da escala, pode-se destacar a não adequação plena do modelo de matriz de dados; entre os indicadores de ajuste, alguns ficaram um pouco abaixo da margem recomenda por Byrne (2010). A homogeneidade da amostra e a prevalência de participantes do sexo masculino podem ser um fator que contribui para esse resultado. Outra limitação desta etapa diz respeito a representatividade da amostra da pesquisa, por essa contar apenas com representantes de uma única unidade federativa. Para contornar ambas as limitações, são sugeridos estudos futuros com amostras de outras regiões do país, além de outras ocupações profissionais e estudos adicionais sobre a estrutura dimensional da medida.

Uma vez que a amostra desta pesquisa também é constituída, em sua maioria, por pessoas de maior escolaridade, as percepções financeiras podem refletir uma realidade restrita (Skinner, 2007) principalmente quanto ao alfabetismo financeiro, que é fundamental para o sucesso nas economias para a aposentadoria (Lusardi, 2015). Sugere-se que futuras pesquisas ampliem o espectro quanto à escolaridade e à renda dos participantes, a fim de que seja possível observar se percepções futuras são influenciadas por variáveis de caráter econômico.

Não foram feitas restrições quanto à idade dos participantes neste estudo a fim de avaliar de forma geral a composição e interação das percepções com demais fatores de contexto. Sugere-se que sequentes trabalhos examinem amostras com participantes acima de 50 anos, visando verificar de modo mais focado se participantes mais próximos à aposentadoria possuem percepções marcadas por essa proximidade temporal (Earl \& Archibald, 2014; Maggiori, Nihil, Froidevaux, \& Rossier, 2013).

A Escala de Percepção de Futuro da Aposentadoria (EPFA) se mostrou adequada para verificar as impressões que os participantes possuem dessa transição, sinalizando correlações com constructos de saúde, centralidade do trabalho e motivos para se aposentar, reforçando a importância de uma abordagem compreensiva para o entendimento da transição para a aposentadoria (Wang \& Shultz, 2010). A escala demonstrou coerência com as principais dimensões gerais ligadas à aposentadoria, cobrindo dimensões de saúde, finanças e relacionamentos, variáveis que têm um papel importante na formação de melhores percepções sobre o futuro (Gutierrez \& Hershey, (2014). Demonstra, assim, a complexidade do fenômeno que envolve mais que o ambiente profissional 
dos trabalhadores, mas também seus arranjos de vida, familiares, de lazer e suas necessidades pessoais de saúde física e mental.

De forma geral, os resultados apresentam que melhores percepções sobre o futuro na aposentadoria relacionaram-se com melhores condições contextuais de vida (saúde geral e saúde no trabalho) além do maior interesse em se aposentar para perseguir desejos pessoais. A centralidade do trabalho aparece como significativa somente para a percepção financeira, demonstrando que o afastamento da vida profissional pode proporcionar melhor vislumbre de futuro financeiro (Selig \& Valore, 2010; Topa, Moriano, \& Moreno, 2012). Ainda que se discuta que a aposentadoria não necessariamente implica o abandono do trabalho, os dados indicam que promover essa quebra das atividades profissionais como centrais na vida pode facilitar o desenvolvimento de um ponto de vista mais positivo quanto ao futuro sem o trabalho (Post, Schneer, Reitman, \& Ogilvie, 2013).

A idade com que os participantes acreditam que se aposentarão se mostrou relevante para as percepções. Acreditar que se aposentará antes da idade mínima exigida (60 anos para mulheres e 65 anos para homens, Brasil, 1995) parece agir como um meio de promover percepções mais positivas sobre a aposentadoria quanto à habilidade para se desligar do trabalho e se manter em relacionamentos de amizade. Esse resultado contraria a expectativa de Desmette e Gaillard (2008) de que menor idade para se aposentar teria correlação com melhores percepções financeiras, conforme estudos com trabalhadores mais velhos dos Estados Unidos. Tal fato pode estar relacionado ao sistema de pensões brasileiro, que se encontra defasado e fornece pouca segurança financeira (Rocha, 2015). Leme e Málaga (2001) pontuam que a população brasileira tende a se aposentar mais jovem, mas que devido a menor renda buscam um meio de complementar a aposentadoria, uma vez que não se sentem seguros para viver somente com a pensão (Jesus, 2015).

Shultz e Wang (2011) indicam que aspectos financeiros e físicos são mais relevantes se as pessoas passam/esperam passar menos tempo aposentadas. Pode-se entender que, na amostra de brasileiros desta pesquisa, é mais importante pertencer a uma rede de suporte e se sentir confortável com a saída do trabalho que sentir-se financeiramente seguro quando pensando na idade para se aposentarem, ligando a aposentadoria precoce a questões mais psicossociais que financeiras, além de questões de longevidade.
Desse modo, optar por se aposentar com idade abaixo da exigida para fins burocráticos serviria ao propósito de contribuir para o melhor ajuste psicossocial dos indivíduos e maior dedicação ao momento da aposentadoria (Curl \& Ingram, 2013; Koposko, Kiso, Hershey, \& Gerrans, 2015).

A diferença entre percepções de desligamento para trabalhadores de empresas públicas e privadas reflete a estrutura diferenciada dos tipos de vínculo empregatício. Profissionais de empresas públicas possuem maior segurança empregatícia e os participantes da presente amostra são em sua maioria professores, profissionais de atendimento ao público em geral e atendimento de emergências, que podem ser consideradas funções desgastantes devido aos contratempos de se lidar com o público, além da elevada burocracia nesse tipo de instituição (Carvalho, Oliveira, \& Silva, 2013). Pode-se acreditar que esses trabalhadores anseiam mais pelo desligamento por essas questões, que impactam em sua satisfação com o trabalho e com a qualidade de vida no trabalho (Henkens \& Leenders, 2010), e que podem torná-los mais susceptíveis à intenção de se desligar, ainda que, por vezes, não se sintam preparados para tal transição psicológica ou financeiramente (Bressan et al., 2013; Juvenal, Viana, Vasconcelos, \& Santos, 2015).

De acordo com as definições previstas na Resolução número002/2003 (CFP, 2003), que expõem os requisitos mínimos para a elaboração de instrumentos psicológicos, a EPFA demonstrou indícios iniciais de que se caracteriza como uma medida confiável para uso em contexto brasileiro, uma vez que apresenta evidências empíricas de validade discriminante quanto à idade esperada para aposentar e o tipo de vínculo empregatício que possuem (público ou privado) e suas propriedades psicométricas correspondem ao esperado segundo prerrogativas da resolução. Segundo Urbina (2007), um instrumento apresenta evidências de validade quando seus resultados empíricos são sustentados pela teoria; a EPFA demonstrou desde sua formulação fatorial boa adequação com a base teórica, mesmo em sua mudança com relação às dimensões da escala. Uma vez que aspectos positivos e negativos fazem parte da vivência de todas as mudanças de fase na vida, e sendo a aposentadoria uma fase que por muitos anos foi tratada como sendo marcada mais por dificuldades que por ganhos, o surgimento de uma escala de Perdas da Aposentadoria mostra a saliência dessa concepção, principalmente ligada ao trabalho e aos relacionamentos. 


\section{Considerações Finais}

Este artigo apresentou o processo de desenvolvimento e validação inicial da EPFA, sinalizando as cinco dimensões que fazem parte da visão compreensiva e holística da aposentadoria, a saber: saúde, relacionamentos, trabalho, estilo de vida e finanças. Ainda que com modificações devido à estrutura fatorial e o surgimento de uma escala de Perdas da Aposentadoria, as cinco dimensões figuraram na versão final da escala e demonstraram serem discriminantes quanto à idade em que os indivíduos esperam se aposentar e o vínculo empregatício que possuem, que são dois relevantes pontos na compreensão do fenômeno da aposentadoria no Brasil, principalmente para a manutenção e viabilidade da Previdência Social.

É importante ressaltar que a amostra pesquisada é composta, em sua maioria, por homens em cargos técnicos especializados e de gestão nas organizações em que atuam. Essa amostra representa uma pequena parcela da realidade brasileira e os resultados podem diferir em populações de maioria feminina ou em cargos operacionais, bem como em população de desempregados. Sugere-se que as próximas pesquisas abordem as diferenças entre essas populações e os dados de saúde e motivação para a aposentadoria a fim de confirmar a relevância dos dados demográficos na percepção futura.

A Escala de Percepção de Futuro da Aposentadoria (EPFA) pode também ser utilizada como forma de corresponder ao primeiro estágio do processo da aposentadoria, que abarca as percepções sobre o evento. Uma vez que expõe percepções ligadas à expectativa de vivência dessa etapa, pode atuar como predecessora de comportamentos de planejamento e decisão de se aposentar. Futuras pesquisas podem utilizar a EPFA a fim de realizar uma avaliação do modelo processual, integrando as variáveis de saúde de saúde física e psicológica além de variáveis de trabalho para compreender a existência de influência e predição entre as etapas do processo da aposentadoria.

Além disso, o entendimento das percepções de futuro é relevante para a construção de planos mais adequados à realidade individual dos trabalhadores. Assim, a EPFA pode ser utilizada como linha de base em programas de planejamento para a aposentadoria, que se baseiam nas expectativas de um envelhecimento bem-sucedido e auxiliam na promoção de uma aposentadoria mais ativa (Dantas \& Oliveira, 2014; Murta et al., 2014).

\section{Referências}

Adams, G. A., \& Rau, B. L. (2011). Putting off tomorrow to do what you want today: Planning for retirement. American Psychologist, 66(3), 180-192. doi:10.1037/a0022131

Beehr, T. A., \& Bowling, N. A. (2012). Variations on a Retirement Theme: Conceptual and Operational Definitions of Retirement. Em M. Wang (Ed.), The Oxford Handbook of Retirement (pp. 42-55). New York, N.Y.: Oxford University Press. doi: 10.1093/ oxfordhb/9780199746521.013.0034.

Brasil. (1995). Lei 9.032 de 28 de abril de 1995. Brasilia: Instituto Nacional de Seguridade Social Recuperado de http://www.planalto.gov.br/ccivil_03/leis/ L9032.htm - art48

Brasil. (2009). Estatuto do Idoso. Brasília: Ministério da Saúde.

Bressan, M., Mafra, S. C. T., França, L., Melo, M. S. D. S., \& Loreto, M. (2013). Bem-estar na aposentadoria: $\mathrm{O}$ que isto significa para os servidores públicos federais. Revista brasileira de geriatria e gerontologia, 16(2), 259-272. doi: 10.1590/S1809-98232013000200006.

Byrne, B. (2010). Structural equation modeling with Amos: Basic concepts, applications and programming (2nd ed.). New York: Routledge, Taylor \& Francis Group.

Carvalho, V. D. d., Oliveira, T. A. d., \& Silva, D. C. d. (2013). Valores organizacionais em instituições públicas brasileiras: Percepções dos servidores em diferentes posições hierárquicas e tipos de entidade da administração indireta. RAM. Revista de Administração Mackenzie, 14(5), 74-103. doi: 10.1590/ S1678-69712013000500004

CFP. (2003). Resolução 002/2003. Brasília, DF. recuperado de http://www2.pol.org.br/legislacao/pdf/ resolucao2003_2.pdf

Curl, A. L., \& Ingram, J. G. (2013). Anticipatory socialization for retirement: A multilevel dyadic model. Clinical Gerontologist, 36(4), 375-393. doi: 10.1080/07317115.2013.788117

Dancey, C. P., \& Reidy, J. (2006). Estatística sem matemática para Psicologia. ( $3^{a}$ edição ed.). Porto Alegre: Artmed.

Dantas, P. M. A. B., \& Oliveira, C. M. (2014). Programas de preparação para a aposentadoria: Desafio 
atual para a gestão de pessoas. Argumentum, 6(1), 116-132. doi: 10.18315/argumentum.v6i1.7473

Desmette, D., \& Gaillard, M. (2008). When a "worker" becomes an "older worker": The effects of age-related social identity on attitudes towards retirement and work. Career Development International, 13(2), 168-185. doi: 10.1108/13620430810860567

Earl, J. K., \& Archibald, H. (2014). Retirement Planning is more than just accumulating resources. European Journal of Management, 14(2), 21-36. doi: 10.18374/ EJM-14-2.2

Fiske, S. T., \& Taylor, S. E. (2013). Social Cognition: From brains to culture (2nd ed.). London: SAGE.

França, L. H. F. P., \& Carneiro, V. L. (2009). Programas de preparação para a aposentadoria: Um estudo com trabalhadores mais velhos em Resende (RJ). Rev. bras. geriatr. gerontol., 12(3), 429-477. Recuperado de http://www.observatorionacionaldoidoso. fiocruz.br/biblioteca/_artigos/150.pdf

França, L. H. F. P., Menezes, G. S., \& Siqueira, A. R. (2012). Planejamento para aposentadoria: A visão dos garis. Rev. bras. geriatr. gerontol., 15(4), 733-745. doi: 10.1590/S1809-98232012000400012

França, L. H. F. P., \& Vaughan, G. (2008). Ganhos e perdas: Atitudes dos executivos brasileiros e neozelandeses frente a aposentadoria. Psicologia em Estudo, 13(2), 207-216. doi: 10.1590/ S1413-73722008000200002

Gutierrez, H. C., \& Hershey, D. A. (2014). Age differences in expected satisfaction with life in retirement. The International Journal of Aging and Human Development, 78(2), 93-114. doi: 10.2190/AG.78.2.a

Henkens, K., \& Leenders, M. (2010). Burnout and older workers' intentions to retire. International Journal of Manpower, 31(3), 306-321. doi: 10.1111/ jonm.12073

Hershey, D. A., \& Henkens, K. (2014). Impact of different types of retirement transitions on perceived satisfaction with life. The Gerontologist, 54(2), 232244. doi: 10.1093 /geront/gnt006

Hira, T. K., Rock, W. L., \& Loibl, C. (2009). Determinants of retirement planning behaviour and differences by age. International Journal of Consumer Studies, 33(3), 293-301. doi: 10.1111/j.1470-6431.2009.00742.x
Hulley, S. B., Cummings, S. R., Browner, W. S., Grady, D., Hearst, N., \& Newman, T. B. (2003). Delineando a pesquisa clínica ( $2^{\mathrm{a}}$ edição ed.). Porto Alegre: Artmed.

Jesus, E. (2015). A previdência social e o trabalhador: Entre o acesso ao direito e a contribuição. Revista Katálysis, 18(2), 213-221. doi: 10.1590/1414-49802015180200007

Juvenal, V. B. S., Viana, D. N. M., Vasconcelos, T. C., \& Santos, J. (2015). Trabalho e aposentadoria: Percepção de professores em situação de pré-aposentadoria. Ciência \& Desenvolvimento, 8(1), 250-272. Recuperado de http://200.223.150.195/revista/ index.php/memorias/article/view/377/231

Kegler, P., \& Macedo, M. M. K. (2015). Trabalho e aposentadoria militar: singularidades de uma travessia psíquica. Psico-USF, 20(1), 25-38. doi: 10.1590/1413-82712015200103

Koposko, J. L., Kiso, H., Hershey, D. A., \& Gerrans, P. (2015). Perceptions of retirement savings relative to peers. Work, Aging and Retirement, 2(1), 65-72. doi: $10.1093 /$ workar/wav019

Leandro-França, C., Murta, S. G., \& Villa, M. B. (2014). Efeitos de uma intervenção breve no planejamento para a aposentadoria. Psicologia: Organizações e Trabalho, 14(3), 257-270. Recuperado de http:// pepsic.bvsalud.org/scielo.php?script=sci_arttext\& pid $=$ S1984-66572014000300002

Lee, W. K. M., \& Law, K. W. K. (2004). Retirement planning and retirement satisfaction: The need for a national retirement program and policy in Hong Kong. Journal of Applied Gerontology, 23(3), 212-233. doi: 10.1177/0733464804268591

Leme, M. C. S., \& Málaga, T. (2001). Entrada e saída precoce da força de trabalho: Incentivos do regime de previdência brasileiro. Revista Brasileira de Economia, 55(2), 205-222. doi: 10.1590/ S0034-71402001000200003

Leung, C. S. Y., \& Earl, J. K. (2012). Retirement resources inventory: Construction, factor structure and psychometric properties. Journal of Vocational Behavior, 81(2), 171-182. doi: 10.1016/j.jvb.2012.06.005

Lusardi, A. (2015). Financial literacy: Do people know the $A B C$ s of finance? Public understanding of science, 24(3), 260-271. doi: 10.1177/0963662514564516 
Macrae, C. N., \& Bodenhausen, G. V. (2000). Social cognition: Thinking categorically about others. Annu. Rev. Psychol., 51, 93-120. doi: 10.1146/annurev.psych.51.1.93

Maggiori, C., Nihil, R., Froidevaux, A., \& Rossier, J. (2013). Development and validation of the transition to retirement questionnaire. Journal of Career Assessment, first published on August 1. doi: 10.1177/1069072713498684

Murta, S. G., Abreu, S., Leandro-França, C., Pedralho, M., Seidl, J., Lira, N. P. M., . . . Gunther, I. A. (2014). Preparação para a aposentadoria implantação e avaliação do programa viva mais! Psicologia: Reflexão e Crítica, 27(1), 1-9. doi: 10.1590/ S0102-79722014000100001

Noone, J. H., Stephens, C., \& Alpass, F. (2010). The process of retirement planning scale (PRePS): Development and validation. Psychol Assess, 22(3), 520-531. doi: 10.1037/a0019512

Noone, J. H., Stephens, C., \& Alpass, F. M. (2009). Preretirement planning and well-being in later life: A prospective study. Research on Aging, 31(3), 295-317. doi: $10.1177 / 0164027508330718$

Paullay, I. M., Alliger, G. M., \& Stone-Romero, E. F. (1994). Construct validation of two instruments designed to measure job involvement and work centrality. Journal of Applied Psychology, 79(2), 224228. doi: apa.org/journals/apl/79/2/224.pdf

Petkoska, J., \& Earl, J. K. (2009). Understanding the influence of demographic and psychological variables on retirement planning. Psychology and Aging, 24(1), 245-251. doi: 10.1037/a0014096

Pires, A. S., Ribeiro, L. V., Souza, N. V. D. O., Sá, C. M. S., Gonçalves, F. G. A., \& Santos, D. M. (2013). A permanência no mundo do trabalho de profissionais de enfermagem com possibilidade de aposentadoria. Ciência, Cuidado e Saúde, 12(2), 338345. doi: $10.4025 /$ cienccuidsaude.v12i2.18298

Post, C., Schneer, J. A., Reitman, F., \& Ogilvie, d. (2013). Pathways to retirement: A career stage analysis of retirement age expectations. Human Relations, 66(1), 87-112. doi: 10.1177/0018726712465657

Rafalski, J. C., \& De Andrade, A. L. (2014). Medidas psicológicas em contexto de aposentadoria. Relatório Preliminar de Pesquisa. Manuscrito não publicado.
Robinson, O. C., Demetre, J. D., \& Corney, R. (2010). Personality and retirement: Exploring the links between the Big Five personality traits, reasons for retirement and the experience of being retired. Personality and Individual Differences, 48(7), 792-797. doi: 10.1016/j.paid.2010.01.014

Rocha, F. R. F. (2015). A previdência social no Brasil: uma política em re-estruturação. Temporalis, 15(30), 453-473. Recuperado de http://periodicos.ufes. br/temporalis/article/view/10943/18405

Selig, A. G., \& Valore, L. A. (2010). Imagens da aposentadoria no discurso de pré-aposentados: Subsídios para a orientação profissional. Cadernos de Psicologia Social do Trabalho, 13(1), 73-87. doi: 10.11606/ issn.1981-0490.v13i1p73-87

Shultz, K. S., Morton, K. R., \& Weckerle, J. R. (1998). The influence of push and pull factors on voluntary and involuntary early retirees' retirement decision and adjustment. Journal of Vocational Behavior, 53(1), 45-57. doi: 10.1006/jvbe.1997.1610

Shultz, K. S., \& Wang, M. (2011). Psychological perspectives on the changing nature of retirement. American Psychologist, 66(3), 170-179. doi: 10.1037/ a0022411

Skinner, J. (2007). Are you sure you're saving enough for retirement? Journal of Economic Perspectives, 21(3), 59-80. doi: 10.1257/jep.21.3.59

Szinovacz, M. (2003). Context and pathways: Retirement as institution, process and experience. Em G. A. Adams \& T. A. Beehr (Eds.), Retirement: Reasons, Processes and Results. (pp. 6-52): Springer Publishing Company.

Topa, G., Moriano, J. A., \& Moreno, A. (2012). Psychosocial determinants of financial planning for retirement among immigrants in Europe. Journal of Economic Psychology, 33(3), 527-537. doi: 10.1016/j. joep.2012.01.003

Trócolli, B. T. (2011). Cognição Social. Em C. V. Torres (Ed.), Psicologia Social - Principais Temas e Vertentes. (pp. 352). Porto Alegre: Artmed.

Urbina, S. (2007). Fundamentos da testagem psicológica. Porto Alegre: Artmed.

Wang, M. (2007). Profiling retirees in the retirement transition and adjustment process: Examining the longitudinal change patterns of retirees' 
psychological well-being. Journal of Applied Psychology, 92(2), 455-474. doi: 10.1037/0021-9010.92.2.455

Wang, M., \& Shultz, K. S. (2010). Employee retirement: A review and recommendations for future investigation. Journal of Management, 36(1), 172-206. doi: 10.1177/0149206309347957

Ware, J. E., \& Sherbourne, C. D. (1992). The MOS 36-item short-form health survey (SF-36). I. Conceptual framework and item selection. Medical Care, 30(6), 473-483. doi: jstor.org/stable/3765916

Zanelli, J. C. (2012). Processos psicossociais, bem-estar e estresse na aposentadoria. Revista Psicologia:
Organizaçõese Trabalho, 12(3),329-340. Recuperadode http://pepsic.bvsalud.org/scielo.php?script=sci_ arttext\&pid=S1984-66572012000300007\&lng=pt $\& \mathrm{t} \operatorname{lng}=\mathrm{pt}$

Zimbardo, P. G., \& Boyd, J. N. (1999). Putting time in perspective: A valid, reliable, individual-differences metric. Journal of Personality and SocialPsychology, 77(6), 1271-1288. doi: 10.1037/0022-3514.77.6.1271

Recebido em: 17/08/2015

Reformulação: 20/01/2016

Aceito em: 16/02/2016

Sobre os autores:

Julia Carolina Rafalski é psicóloga e Mestre em Psicologia pela Universidade Federal do Espírito Santo. Realizou estágio científico na University of Sydney (Austrália) com bolsa FAPES para estudar processos de aposentadoria e trabalho para indivíduos mais velhos. Tem interesse na área de carreira para trabalhadores mais velhos, aposentadoria, preconceito contra a idade e conflito trabalho-família.

E-mail: jcrafalski@gmail.com

Alexsandro Luiz De Andrade é professor e pesquisador do campo da Avaliação Psicológica e Carreira da Universidade Federal do Espírito Santo. Pós Doutor em Psicologia Social e Personalidade pela Universidade do Hawaii. Possui interesse de pesquisa e intervenção no campo de Métodos Quantitativos de Pesquisa, Carreira, Avaliação Psicológica e Fatores Humanos no Trabalho.

E-mail: alexsandro.deandrade@yahoo.com

Contato com os autores:

Programa de Pós-Graduação em Psicologia

Av. Fernando Ferrari, 514, Goiabeiras

Vitória-ES, Brasil

CEP: 29075-910 